\title{
A predominant $\beta$-CGTase G1 engineered to elucidate the relationship between protein structure and product specificity.
}

\begin{abstract}
Low reaction yields and the high cost of obtaining a single type of pure CD make $\gamma-C D$ costly. Using rational design and with the aid of 3D modeling structures, recombinant CGTase from Bacillus sp. G1 was molecularly engineered with the aim of producing a higher percentage of $\gamma$-CD. A single mutation at subsite -3 , denoted H43T, was found to increase $\gamma$ CD production from $10 \%$ to approximately $39 \%$ using tapioca starch. This novel increment was probably the result of reduced steric hindrance to the formation of $\gamma$-CD because of the shortened side chain together with the shortened loop at positions 86-89, at substrate-binding subsite -3 . A mutation (Tyr188 $\rightarrow$ Trp) and a deletion at loop 139-144 showed little effect on product specificity; however, mutagenesis at these sites affected cyclization, coupling and hydrolysis activities as well as the kinetic properties of the mutant CGTase. Based on rational design, three further mutations of the mutant H43T (denoted H43T/A(139144)/S134T/A137V/L138D/V139I, H43T/S85G and H43T/Y87F) were constructed and produced $\gamma$-CD with yields of $20 \%, 20 \%$ and $39 \%$, respectively. The mutant H43T/ $\Delta(139$ 144)/S134T/A137V/L138D/V139I had very low cyclization and coupling activities, however their hydrolysis activity was retained. Double mutation (H43T/S85G) caused the enzyme to exhibit higher starch hydrolysis activity, approximately 26 times higher than the native CGTase G1. Although the mutants $\mathrm{H} 43 \mathrm{~T}$ and $\mathrm{H} 43 \mathrm{~T} / \mathrm{Y} 87 \mathrm{~F}$ could produce the same percentage $(39 \%)$ of $\gamma-C D$, the latter was more efficient as the total amount of CD produced was higher based on the Vmax and kcat values.
\end{abstract}

Keyword: CGTase; Cyclodextrin; Product specificity; Protein engineering. 